\title{
Ab in den Ausguß?
}

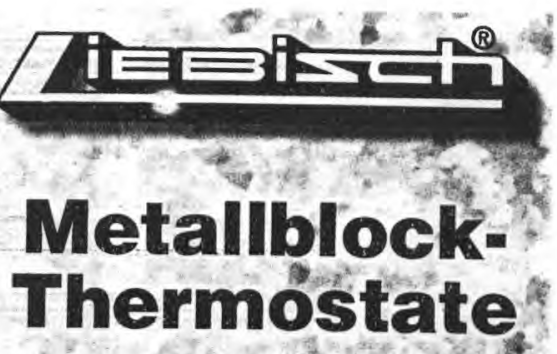

Die Entsorgung von Stoffen über das $A b$ wasser ist äußerst bequem, deshalb ist die Versuchung groß, möglichst viel auf diese Art loszuwerden. Dabei wird jedoch die Reinigungsleistung der Kläranlage maßlos überschätzt. Hier wird ein Beurteilungssystem vorgestellt, mit dem die gewässerökologische Verantwortung bei der Entsorgung von Stoffen über das Abwasser auf einfache Art wahrgenommen werden kann.

Abfälle einfach in den Ausguß schütten nichts macht weniger Mühe als diese Art der Entsorgung. Die Kläranlage wird's schon wieder richten. Diese Annahme ist aber leider ein Irrglaube, der zu ernsthaften Problemen sowohl in der Klärananlage als auch in der Umwelt führen kann. Umgekehrt ist es nicht nötig, deshalb gleich das Kind mit dem Bade auszuschütten und jedweden flüssigen Abfall als aufwendig zu entsorgenden Sondermüll zu betrachten. Ein pragmatisches Beurteilungssystem - basierend auf den Ökodaten des Sicherheitsdatenblatts und der Kenninis der involvierten Abwasserinfrastruktur - für die Entscheidung Wegschütten - ja oder nein? kann das Chemiker-Lehen leichter machen.

\section{Was kann eine Kläranlage?}

In der Schweiz werden über $95 \%$ der anfallenden Abwässer in Kläranlagen (Abbildung 1) behandelt. Diese Anlagen enthalten mindestens eine mechanische und eine biologische Reinigungsstufe. Die Aufgabe der mechanischen Stufe ist es, wasserunlösliche Stoffe aus dem Abwasser zu entfernen. Da die meisten unlöslichen Abwasserinhaltsstoffe eine größere Dichte besitzen als das Wasser, sedimentieren sie im Vorklärbecken, können abgetrennt und als sogenannter Primärschlamm dem Faulraum zugeleitet werden. Wasserlösliche Stoffe durchfließen das Vorklärbecken. Es ist Aufgabe der biologischen Reinigungsstufe, diese Stoffe aus dem Abwasser zu entfernen. Diese Arbeit wird von den unzähligen Mikroorganismen vollbracht, die sich im Belebungsbecken befinden (Abbildung 2). Sie können jedoch nur dann mit Erfolg arbeiten, wenn die Abwasserinhaltsstoffe Nahrung für diese Kleinlebewesen darstellen, das heißt, wenn die Stoffe aerob biologisch abbaubar sind. Dieser aerobe biologische Abbau benötigt Sauerstoff, deshalb muß das Belebungsbecken intensiv belüftet werden.

Gelangen wasserlösliche, bakterientoxische Substanzen ins Abwasser, können die Mikroorganismen der biologischen Stufe geschädigt werden. Dadurch wird die Reinigungsleistung beeinträchtigt - solche Stoffe sind daher im Abwasser zwingend zu vermeiden. Nicht selten verschwinden Stoffe in der biologischen Reinigungsstufe durch reine Adsorption an die riesige Oberfläche der Kleinlebewesen aus dem Wasser. Derartige Sub-

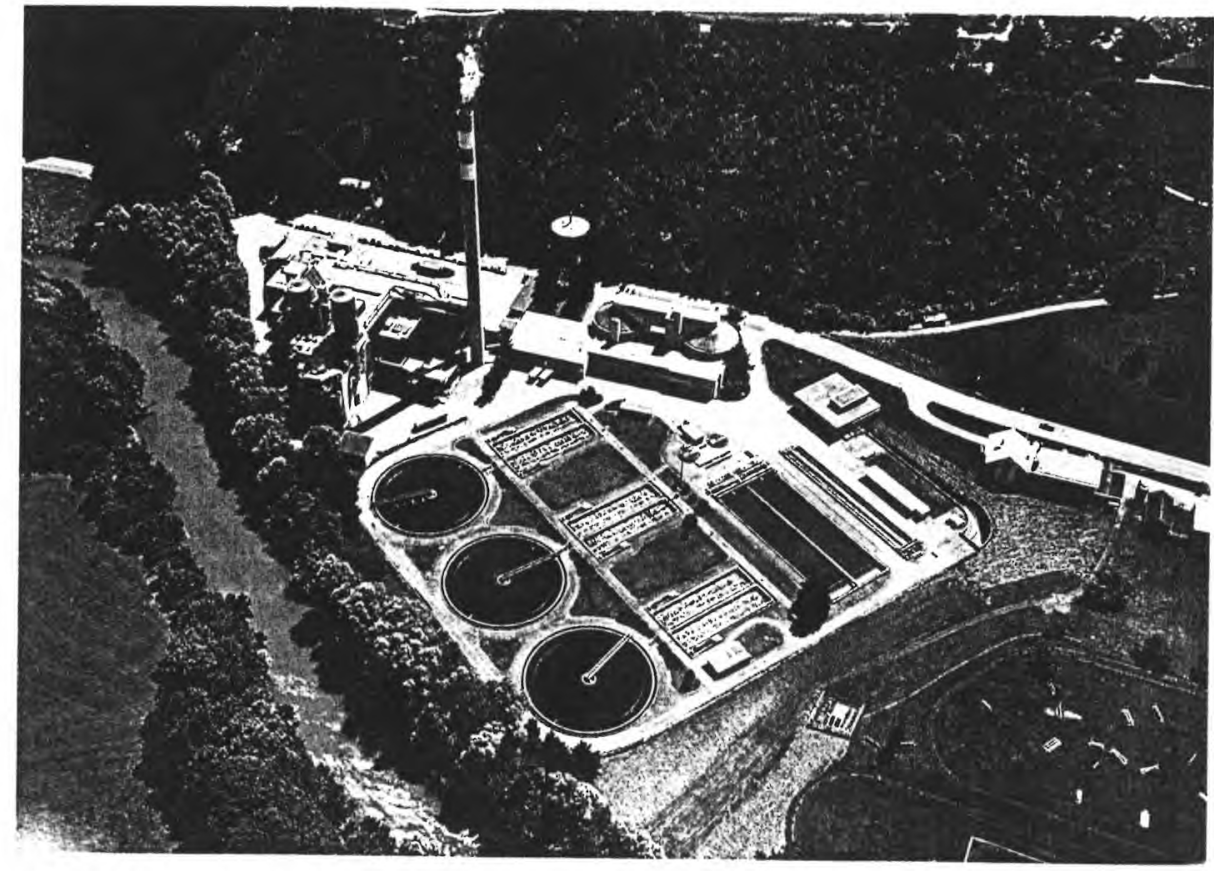

Abb. 1. Abwasserreinigungsanlage mit Kehrichtverbrennung: Bruggen, St. Gallen/ Schweiz. 

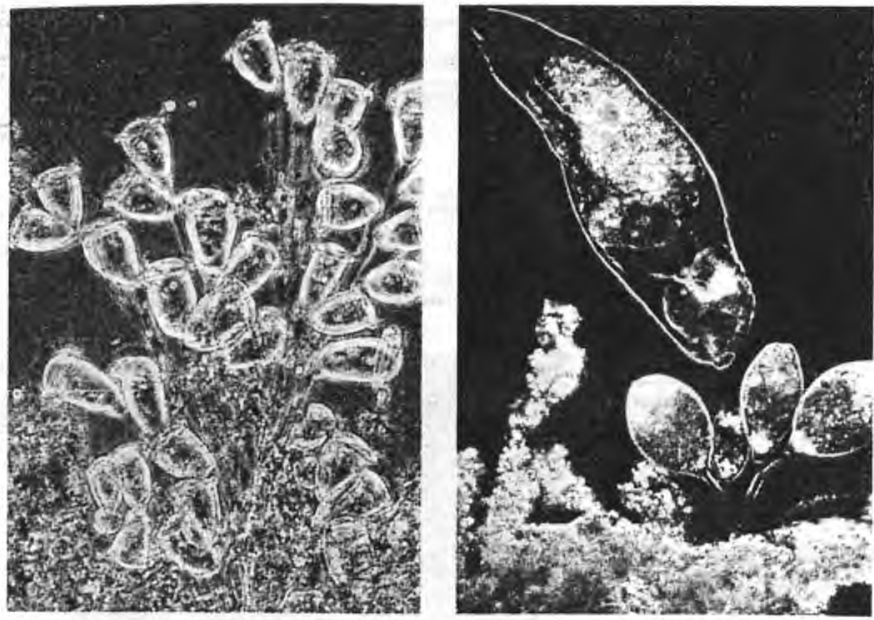

\section{Abb. 2. Mikro- organismen des Belebtschlamms: Glockentierchen, Rädertierchen.}

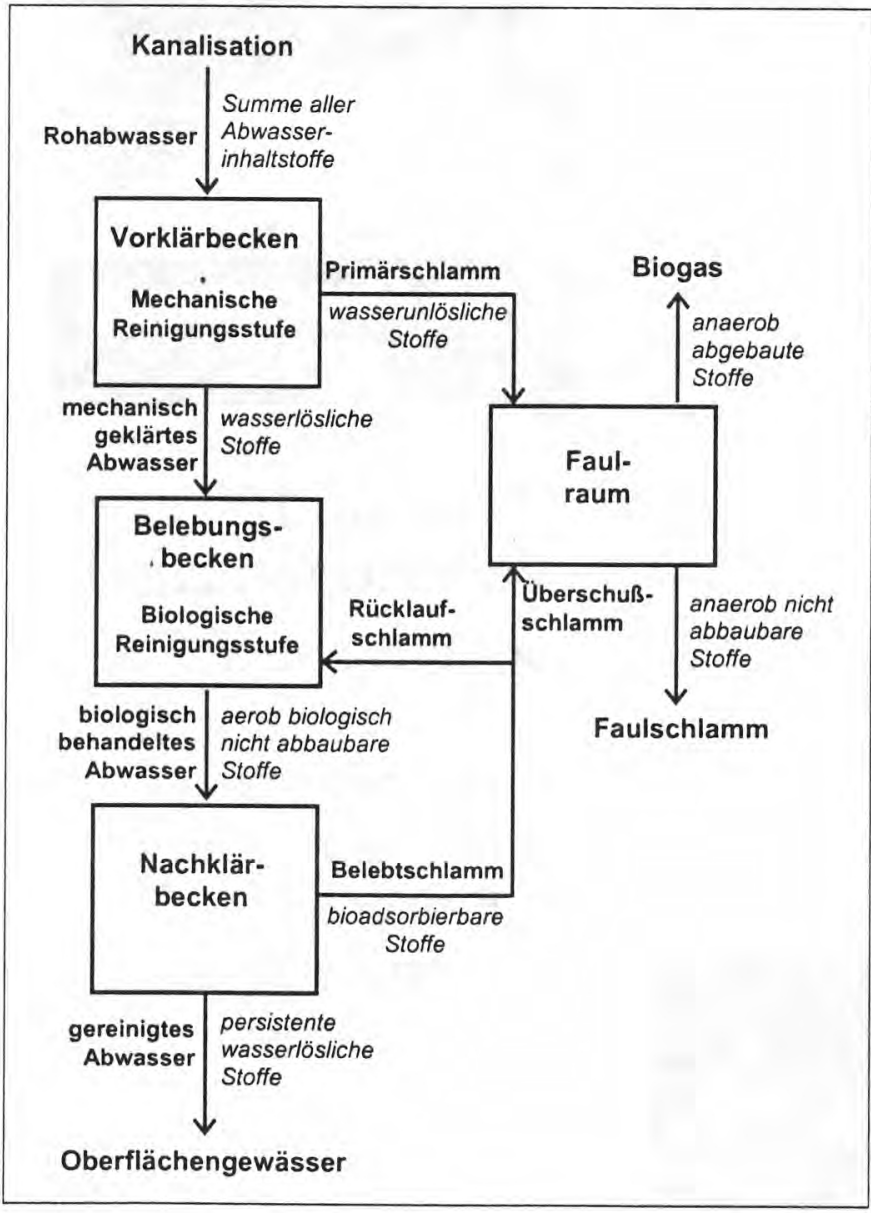

stanzen Laborexperimente durchführen bzw. in Auftrag geben. In diesen Experimenten wird die $\mathrm{zu}$ beurteilende Umweltsituation simuliert, um die Wirkung oder Verhaltensweise der Testsubstanz zu erkennen. Da die gewählten Versuchsbedingungen die Resultate beeinflussen können, ist es für vergleichende Stoffbeurteilungen notwendig, daß mit normierten Untersuchungsverfahren gearbeitet wird. Die OECD (Oganisation for Economic Cooperation and Development) stellt für diesen Zweck eine Methodensammlung zur Verfügung. ${ }^{1)}$

\section{Belebtschlammtoxizität}

Ein weit verbreiteter Test für die Beurteilung der Belebtschlammtoxizität ist der OECDTest 209: Die zu testende Substanz wird gelöst und mit Belebtschlamm aus der Kläranlage und leicht abbaubaren Stoffen gemischt. Da die aeroben Mikroorganismen des Belebtschlamms günstige Nahrungsbedingungen vorfinden, setzt, sofern die Testsubstanz nicht giftig ist, ein intensiver Stoffwechsel ein, der von einer Sauerstoffzehrung begleitet ist. Über die Messung der Sauerstoffzehrung kann somit die Belebtschlammtoxizität der Testsubstanz in Erfahrung gebracht werden. Diejenige Testsubstanzkonzentration, bei der die Sauerstoffzehrung um $50 \%$ vermindert wurde, wird als $\mathrm{EC}_{50}$ (EC = effektive Konzentration) bezeichnet. Je geringer der gefundene Wert, desto gravierender die Toxizität.

\section{Bioelimination und aerober biologischer Abbau}

Die Mikroorganismen der Kläranlage entfernen die Stoffe aus dem Abwasser zum einen durch Abbau und zum anderen durch Adsorption (Abbildung 4). Um diese Parameter zu bestimmen, gibt es verschiedene von der OECD normierte Methoden. Tabelle 2 stellt die wichtigsten zusammen.

Bei allen Testverfahren wird die in Wasser gelöste Testsubstanz mit Mikroorganismen in Kontakt gebracht und genügend Sauerstoff
Abb. 3. Abwasserreinigungsanlage im Überblick. stanzen werden mit dem Belebtschlamm im Nachklärbecken abgetrennt und im Überschußschlamm dem Faulraum zugeführt. Die vereinigten Schlämme, der Primärschlamm und der Überschußschlamm, gelangen in den unter Sauerstoffausschluß stehenden Faulraum. Er stellt den Lebensraum anaerober Mikroorganismen dar. Unter diesen Bedingungen können anaerob abbaubare Stoffe zu Biogas abgebaut werden. Das ausgefaulte Produkt, der Faul- oder Klärschlamm, wird in der Schweiz zu ca. $50 \%$ als Dünger in der Landwirtschaft plaziert respektive zu ca. $50 \%$ getrocknet und verbrannt. Abbildung 3 gibt einen Überblick über die verschiedenen Stufen einer mechanisch-biologischen Abwasserreinigungsanlage.

\section{Kläranlagentauglich oder nicht?}

Um die Verhaltensweise von Stoffen in Kläranlagen beurteilen zu können, ist eine ganze Reihe von Informationen notwendig. Sie verbergen sich zum Großteil in den ökologischen Stoffdaten der entsprechenden EUProduktsicherheitsblätter. Diẹ entscheidenden Fragen sind in Tabelle 1 zusammengefaßt. Daneben muß die lokale Abwasserinfrastruktur berücksichtigt werden. Das in Abbildung 5 dargestellte Fließschema führt anhand der gegebenen Antworten zu einer Klassifizierung der betrachteten Substanz, so daß eine Aussage über ihre „Kläranlagenverträglichkeit" getroffen werden kann. Zur Beantwortung der in Tabelle 1 gestellten Fragen muß der Inverkehrbringer von Subzur Verfügung gestellt. Über die Bestimmung des gelösten organischen Kohlenstoffs (DOC) kann eine Aussage über die Bioelimination bzw. den Bioabbau gemacht werden: Je nach der eingesetzten Konzentration der Mikroorganismen ist nur mit aerobem Bioabbau oder auch mit Bioadsorption zu rechnen. Entweder anhand des biochemischen Sauerstoffbedarfs (BSB) oder anhand der Kohlendioxidemission kann die Mineralisation ermittelt werden, die Rückschlüsse auf den Bioabbau zuläßt. Bioelimination und Mineralisation können - wenn man Untersuchungskosten sparen möchte - auch in einem Experiment bestimmt werden. ${ }^{2)}$

\section{Anaerobe biologische Abbaubarkeit und Toxizität}

Dem anaeroben biologischen Abbau wurde bis heute viel weniger Beachtung geschenkt als dem aeroben. Erste Normen sind jedoch 
verfügbar., Allen Verfahren ist gemeinsam. $\mathrm{daB}$ die in Wasser gelöste Testsubstanz in $\mathrm{Ab}$ wesenheit von Sauerstoff mit Faulschlamm zusammengebracht und die Biogasbildung über das Biogasvolumen oder den Biogasdruck gemessen wird. Daß derartige Untersuchungen nicht kompliziert sein müssen. zeigen Arbeiten von Baumann:4) mit seinem Verfahren kann auch die Toxizität für Anaerobier einfach bestimmt werden.

\section{Die Infrastruktur der Kläranlage}

Viele Kläranlagen sind aus Kapazitätsgründen bei starken Niederschlägen überfordert. Um diesen Situationen zu begegnen, befinden sich im Einlauf häufig sogenannte Hochwasserentlastungen. Es handelt sich dabei um einfache Überläufe, die bei einem Hochwasserereignis das unbehandelte Abwasser direkt dem Vorfluter zuleiten. Unter diesen Bedingungen können wasserunlösliche und wasserlösliche Abwasserinhaltsstoffe direkt in das Oberflächengewässer gelangen. Dadurch können Stoffe, die im Normalbetrieb in der Kläranlage entfernt werden, die aquatischen Organismen unserer Gewässer erreichen. Stoffe mit hoher Toxizität für Wasserorganismen werden dann zu einem Problem. Um dieser unerfreulichen Situation zu begegnen. werden immer mehr Abwasserreinigungsanlagen mit Hochwasserrückhaltebekken ausgestattet. In riesigen Becken wird das Wasser gespeichert und kann anschließend in der Kläranlage behandelt werden.

Ein weiterer Punkt, der beachtet werden muß, ist das Schicksal des Faulschlamms. Wird er landwirtschaftlich genutzt, deponiert oder getrocknet und verbrannt? Organische Stoffe, die in den Faulschlamm gelangen, werden bei der Schlammverbrennung mineralisiert. Bei der landwirtschaftlichen Nutzung oder Deponierung dagegen kontaminieren sie die entsprechenden Lebensräume. Höchst unerfreulich ist die Situation dann, wenn landwirtschaftlich genutzte Schlämme
Tabelle 1. Schema zur Beurteilung des Verhaltens von Stoffen in Kläranlagen.

\begin{tabular}{|c|c|c|}
\hline Frage & Antwort & Konsequenz \\
\hline 1. Ist der Stoff wasserlöslich? & nein & $\begin{array}{l}\text { er durchfließt die mechanische Stufe und } \\
\text { gelangt in das Belebungsbecken - weiter mit } \\
\text { Nr. } 2 \\
\text { er sedimentiert im Vorklärbecken und wird im } \\
\text { Primärschlamm dem Faulraum zugeführt - } \\
\text { weiter mit Nr. } 5\end{array}$ \\
\hline $\begin{array}{l}\text { 2. Ist der wasserlösliche Stoff } \\
\text { toxisch für den } \\
\text { Belebtschlamm? }\end{array}$ & ja & $\begin{array}{l}\text { es kann zu einer Vergiftung der biologischen } \\
\text { Stufe kommen } \\
\text { es ist mit keiner Beeinträchtigung des } \\
\text { Belebtschlamms zu rechnen - weiter mit Nr. } 3\end{array}$ \\
\hline $\begin{array}{l}\text { 3. Wird der wasserlösliche } \\
\text { Stoff durch den } \\
\text { Belebtschlamm aus dem } \\
\text { Wasser eliminiert? }\end{array}$ & nein & $\begin{array}{l}\text { der Stoff wird entweder biologisch abgebaut } \\
\text { oder an den Belebtschlamm adsorbiert - } \\
\text { weiter mit Nr. } 4 \\
\text { der Stoff verbleibt im gereinigten Abwasser - } \\
\text { Risikoabschätzung erforderlich }\end{array}$ \\
\hline $\begin{array}{l}\text { 4. Ist der wasserlösliche Stoff } \\
\text { aerob biologisch abbaubar? }\end{array}$ & ja & $\begin{array}{l}\text { der Stoff verschwindet in der biologischen } \\
\text { Stufe durch Bioabbau } \\
\text { der Stoff verbleibt im Wasser oder wird an } \\
\text { den Belebtschlamm adsorbiert - } \\
\text { Risikoabschätzung oder weiter mit Nr. } 6\end{array}$ \\
\hline $\begin{array}{l}\text { 5. Ist der wasserunlösliche } \\
\text { Stoff anaerob biologisch }\end{array}$ & $\begin{array}{l}\text { ja } \\
\text { nein }\end{array}$ & $\begin{array}{l}\text { er wird in Biogas umgewandelt } \\
\text { er verbleibt im Klärschlamm }\end{array}$ \\
\hline
\end{tabular}

\begin{tabular}{lll}
$\begin{array}{l}\text { 6. Ist der wasserunlösliche } \\
\text { Stoff toxisch für Anaerobier? }\end{array}$ & $\begin{array}{l}\text { ja } \\
\text { nein }\end{array}$ & $\begin{array}{l}\text { er kann die Anaerobier im Faulturm schädigen } \\
\text { die Anaerobier werden nicht geschädigt }\end{array}$ \\
\hline
\end{tabular}

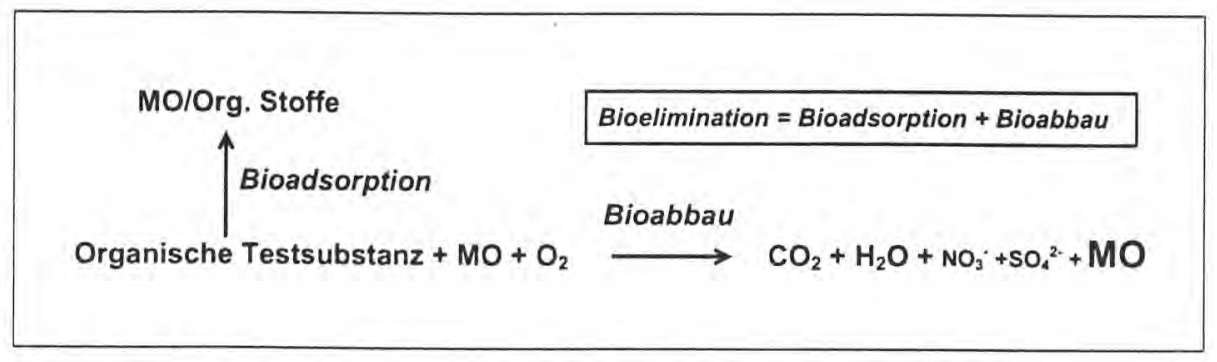

Abb. 4. Die Bioelimination setzt sich aus Bioadsorption und Bioabbau zusammen. MO = Mikroorganismen .

\section{Tabelle 2. Überblick über die Untersuchung der Bioelimination und des Bioabbaus.}

\begin{tabular}{|c|c|c|c|c|}
\hline Bestimmungsgröße & Bioelimination & Bioabbau (Totalabbau) & Mineralisation $^{\text {b) }}$ & Mineralisation $^{\text {b) }}$ \\
\hline Untersuchungsparameter & $\begin{array}{l}\text { DOC } \\
\text { gelöster organischer } \\
\text { Kohlenstoff }\end{array}$ & $\begin{array}{l}\text { DOC } \\
\text { gelöster organischer } \\
\text { Kohlenstoff }\end{array}$ & $\begin{array}{l}\text { BSB (englisch BOD) } \\
\text { biochemischer } \\
\text { Sauerstoffbedarf }\end{array}$ & Kohlendioxidemission \\
\hline $\begin{array}{l}\text { Eingesetzte } \\
\text { Mikroorganismen- }\end{array}$ & hoch & gering & gering & gering \\
\hline
\end{tabular}

Mikroorganismen-

Konzentration

\section{OECD-Methode \\ Bezeichnung}

Günstige Beurteilung von

\section{A SCAS-Test}

302 B Mod. Zahn-Wellensoder EMPA-Test

303 A Coupled-Units-Test

\author{
301 E Mod. Screening- \\ Test \\ 301 C Mod. MITI-Test \\ 301 D Closed Bottle Test \\ 301 F Respirationstest
}

301 B Mod. Sturm-Test

\footnotetext{
Stoffen wenn:
}

a)

$>70 \%$

$>60 \%$

$>60 \%$

a) Hohe Werte zeigen, daß die Kläranlage den Stoff aus dem Abwasser zu entfernen vermag. Es kann aber nicht zwischen Bioadsorption und Bioabbau differenziert werden. Praktiker gehen häufig von $80 \%(302 \mathrm{~A}$ und B) bzw. $75 \%(303 \mathrm{~A})$ aus.

b) Auch vollständig abbaubare Stoffe werden nie zu $100 \%$ mineralisiert. Mit einer Inkorporation von $20 \%$ in die Biomasse muß gerechnet werden. Die Faustregel besagt: Bioabbau $\approx 1.25 \times$ Mineralisation. 


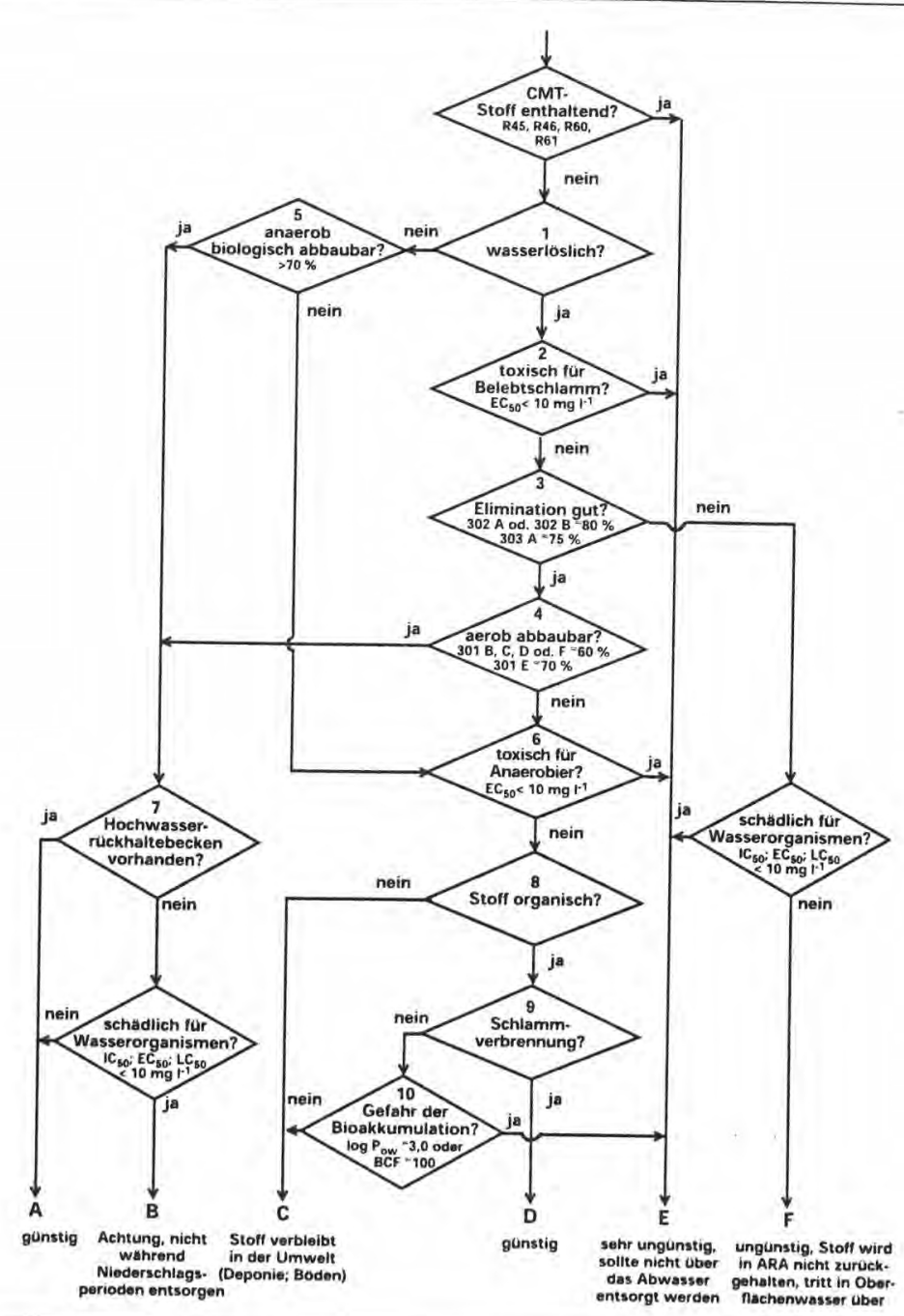

Abb. 5. Fließschema zur Klassifizierung von Substanzen hinsichtlich ihrer „Kläranlagenverträglichkeit".

\section{Tabelle 3. Bedeutung der gewässerökologischen Stoffklassen.}

\begin{tabular}{ll} 
Klasse & Charakterisierung des Stoffs \\
\hline A & $\begin{array}{l}\text { Stoffe, die in der ARA durch biologischen } \\
\text { Abbau vollständig verschwinden. }\end{array}$ \\
B & $\begin{array}{l}\text { Stoffe, die in der ARA durch biologischen } \\
\text { Abbau vollständig verschwinden. Sie } \\
\text { besitzen als solche eine hohe aquatische } \\
\text { Toxizität. }\end{array}$
\end{tabular}

C Anorganische Stoffe, die in den Faulschlamm übertreten. Bei landwirtschaftlicher Nutzung werden sie diffus verteilt.

D Organische Stoffe, die in den Faulschlamm gelangen und durch die Verbrennung mineralisiert werden.

E Diese Klasse enthält die unerfreulichsten Stoffe. Entweder sind sie cancerogen, mutagen, teratogen, toxisch für Aerobier. Anaerobier, aquatische Organismen oder bioakkumulierbar.

F Stoffe, die im gereinigten Abwasser verbleiben und somit in den aquatischen Lebensraum gelangen.
Stoffe enthalten, die zur Bioakkumulation neigen. Die Tendenz der Stoffe, sich in Organismen anzureichern, kann einfach durch die Bestimmung des Octanol/Wasser-Verteilungskoeffizienten abgeschätzt werden. ${ }^{5.6)}$

\section{Stoff-Klassifizierung}

Mit dem in Abbildung 5 vorgestellten Entscheidungsbaum gelingt es, anhand der ökologischen Stoffdaten (Antworten auf die Fragen der Tabelle 1) und unter Berücksichtigung der vorhandenen Abwasserinfrastruktur, Stoffe gewässerökologisch in sechs Klassen ( $A$ bis $F$ ) einzuteilen (Tabelle 3).

Die ökologischen Stoffdaten können dem EU-Sicherheitsdatenblatt entnommen werden. Cancerogene, mutagene und teratogene Stoffe (CMT-Stoffe), die mit den R-Sätzen R 45 (kann Krebs erzeugen), R 46 (kann vererbbare Schäden verursachen), R 60 (kann die Fortpflanzungsfähigkeit beeinträchtigen) und R 61 (kann das Kind im Mutterleib schädigen) auf den Sicherheitsdatenblättern gekennzeichnet sind, gelangen automatisch in die risikoreichste Klasse E.

Leider fehlen auf heutigen Sicherheitsdatenblättern meistens Informationen zum anaeroben biologischen Abbau und zur Anaerobiertoxizität. Dies wird sich hoffentlich in $\mathrm{Zu}$ kunft ändern. In einer Übergangszeit ist es aus statistischen Gründen sinnvoll, diese Stoffe als anaerob biologisch nicht abbaubar und anaerob nicht toxisch zu behandeln.

In Tabelle 4 sind drei Beispiele für eine Stoffklassifizierung zusammengestellt. Die entsprechenden farbig markierten Pfade sind im Entscheidungsbaum (Abbildung 6) zu verfolgen.

\section{Risikoabschätzungen in kritischen Fällen}

Verhaltensempfehlung

Können über das Abwasser entsorgt werden.

Können über das Abwasser während niederschlagsfreier Zeiten entsorgt werden. Während Niederschlagsperioden nicht entsorgen.

Nur inerte Stoffe bedenkenlos über das Abwasser entsorgen.

Können über das Abwasser entsorgt werden.

Solche Stoffe sollten nicht über das Abwasser entsorgt werden.

Die Entsorgung solcher Stoffe ist nur nach sorgfältigen Risikobetrachtungen zu verantworten.
Substanzen, die durch Kläranlagen hindurchtreten, d.h. im gereinigten Abwasser immer noch vorhanden sind, gelangen zwangsläufig in den Vorfluter. In unserem Klassifizierungsschema (Abbildung 5) sind es die Stoffe der Klasse F. In diesen Fällen ist eine Risikobewertung vorzunehmen (Beispiel 3 in Tabelle 4). Solche Bewertungen beginnen mit der Abschätzung der Stoffkonzentration im Vorfluter. Dafür muß die pro Zeiteinheit über das Abwasser entsorgte Stoffmenge in Erfahrung gebracht werden. Der Klärwärter der zuständigen Abwasserreinigungsanlage ist in der Lage, die pro Zeiteinheit anfallende Menge geklärten Abwassers und das Verdünnungsverhältnis, mit dem im Vorfluter gerechnet werden kann, abzuschätzen. Anhand dieser Daten ist es möglich, eine voraussichtliche Stoffkonzentration im Vorfluter (PEC $=$ Predicted Environmental Concentration) anzugeben.

Ausgehend von ökologischen Dosis-Wirkungs-Daten für aquatische Organismen (Fische, Daphnien, Algen, Bakterien etc.) wird nun versucht. die PEC zu beurteilen. Dies gelingt um so besser, je fundierter die aquatischen Toxizitätsdaten sind. Da meistens le- 


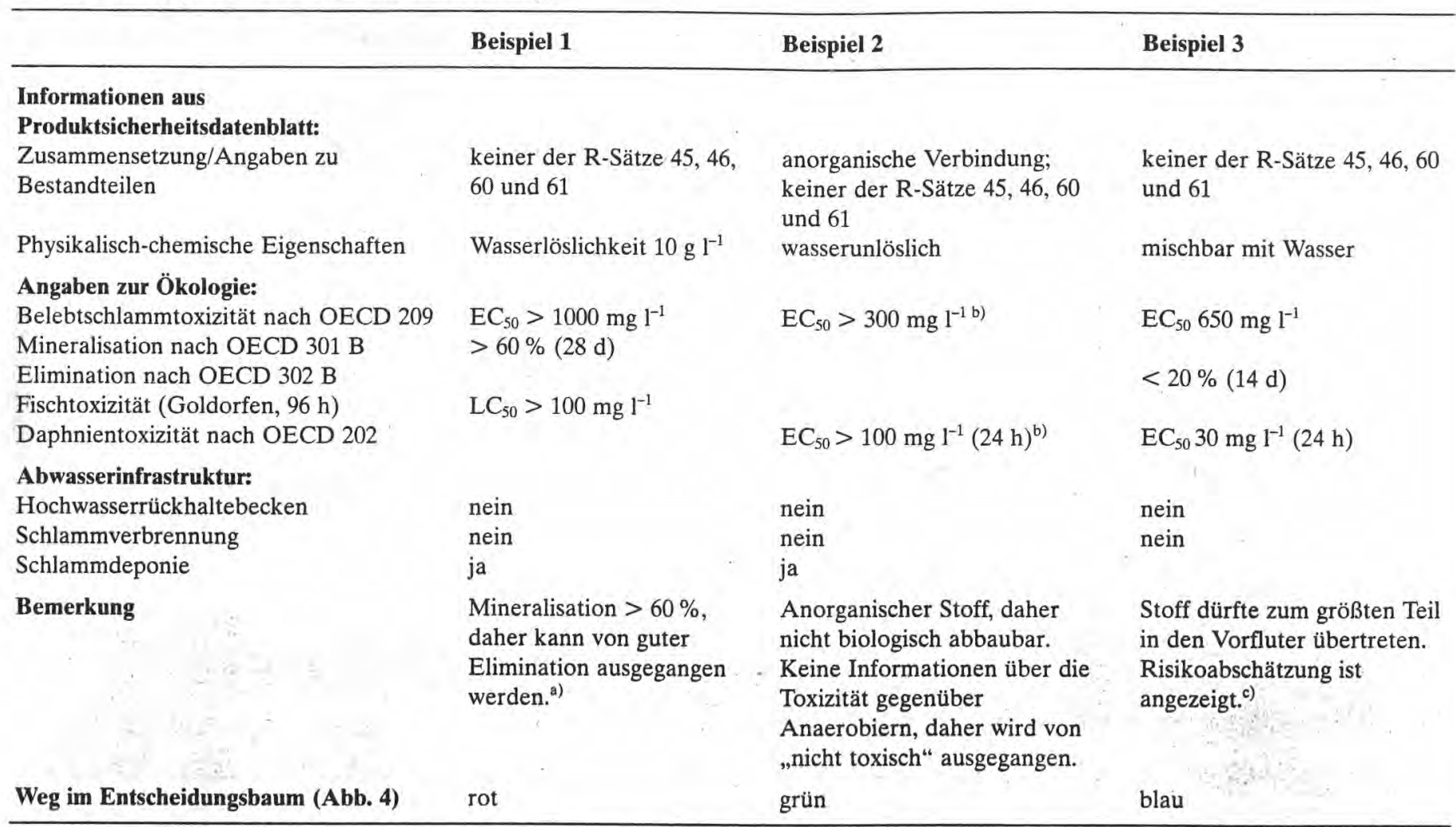

a) Bei der Elimination ist der in die Biomasse inkorporierte Anteil enthalten, deshalb ist der Grenzwert dort für die 302 -Verfahren $\geq 80 \%$.

b) An der Suspension untersucht.

c) Folgende Daten wurden erhoben: Verbrauch des Stoffs $650 \mathrm{~kg} \mathrm{~d}^{-1}$, davon gelangen $40 \%$ ins Abwasser. Daten zur Kläranlage: typische Abwassermenge $9000 \mathrm{~m}^{3} \mathrm{~d}^{-1}$; typisches Verdünnungsverhältnis im Vorfluter 1:20. Daraus kann das Risiko wie folgt abgeschätzt werden:

Stoffkonzentration in der Kläranlage: $650000000 \mathrm{mg} \mathrm{d}^{-1} \times 40 \% / 9000000 \mathrm{l} \mathrm{d}^{-1}=29 \mathrm{mg} \mathrm{l}^{-1}$

Die Belebtschlammtoxizität beträgt: $\mathrm{EC}_{50} 650 \mathrm{mg} \mathrm{l}^{-1}$. Somit dürften die $29 \mathrm{mg} \mathrm{l}^{-1}$ die biologische Reinigungsstufe nicht stören.

Im Vorfluter ist mit einer 20fach geringeren Konzentration zu rechnen, PEC somit ca. $1,5 \mathrm{mg} \mathrm{l}^{-1}$.

Für PNEC gilt: Sicherheitsfaktor $=1000$, da nur ein akuter Toxizitätswert vorhanden; $30 \mathrm{mg} \mathrm{l}^{-1} / 1000=0,03 \mathrm{mg} \mathrm{l}^{-1}$.

Der Vergleich PEC mit PNEC zeigt, daß das Risiko zu groß ist. Das Produkt darf somit nicht ohne Abwasservorbehandlung in derart großen Mengen dem Abwasser übergeben werden.

\section{Die neuen Media-}

\section{Informationen} für 1999 sind da!

Fordern Sie diese gleich schriftlich oder mündlich an...

Ihre Anzeigenabteilung:

Telefon: +49 (0) 6201 - 606-565

Telefax: +49 (0) 6201 - 606-550

E-mail: MSchulz@wiley-vch.de
Tabelle 5. Von der OECD vorgeschlagene Sicherheitsfaktoren.

\begin{tabular}{ll}
\hline Vorhandene Daten & $\begin{array}{l}\text { Sicherheits- } \\
\text { faktor }\end{array}$ \\
\hline $\begin{array}{l}\text { Akute } \text { LC }_{50} \text {-Werte für ein } \\
\text { oder zwei aquatische } \\
\text { Organismen }\end{array}$ & 1000 \\
$\begin{array}{l}\text { Akute LC } \text { L }_{50} \text {-Werte für Fische, } \\
\text { Krustentiere und Algen }\end{array}$ & 100 \\
$\begin{array}{l}\text { Chronische NOEC-Werte } \\
\text { für Fische, Krustentiere und } \\
\text { Algen }\end{array}$ & 10 \\
\hline
\end{tabular}

diglich rudimentäre Informationen vorliegen, versucht man mit Hilfe von Sicherheitsfaktoren der Situation adäquat zu begegenen. In Tabelle 5 sind die je nach Datenlage üblichen Sicherheitsfaktoren zusammengestellt.

Konkret wird die gravierendste Toxizitätsangabe (die minimale toxisch wirkende Dosis aus den vorhandenen Dosis-Wirkungs-Daten) durch den entsprechenden empfohlenen $\mathrm{Si}$ cherheitsfaktor dividiert. Die resultierende Stoffkonzentration sollte dann mit großer Wahrscheinlichkeit keine Effekte mehr auslösen $($ PNEC $=$ Predictet No Effect Concen- tration). Liegt die voraussichtliche Konzentration im Vorfluter PEC unterhalb der PNEC, besteht voraussichtlich kein Gefahrenpotential (Abbildung 7).

\section{Fazit}

Basierend auf den Angaben in einem guten Sicherheitsdatenblatt und der Kenntnis der involvierten Abwasserinfrastruktur ist es relativ einfach möglich, die Umwelt vor gewässerökologisch ungünstigen Stoffen zu schützen.

Urs Baumann, St.Gallen/Schweiz

1) OECD, Guidelines for Testing of Chemicals, 1993.

2) U. Baumann, M. Abele, E. Pletscher, M. Siegfried, Tenside Surf. Det. 1997, 34, 336.

3) ISO 11734, 1995: Water quality - Evaluation of the "ultimate" anaerobic biodegradability of organic compounds in digested sludgeMethod by measurement of the biogas production.

4) U. Baumann, Gas Wasser Abwasser (GWA) 1997, 77, 164.

5) OECD Method 117, 1989.

6) L.O. Renberg, S.G. Sundström, A.C. Rosén, Toxicology and Environmental Chemistry, 1985, 10, 333. 


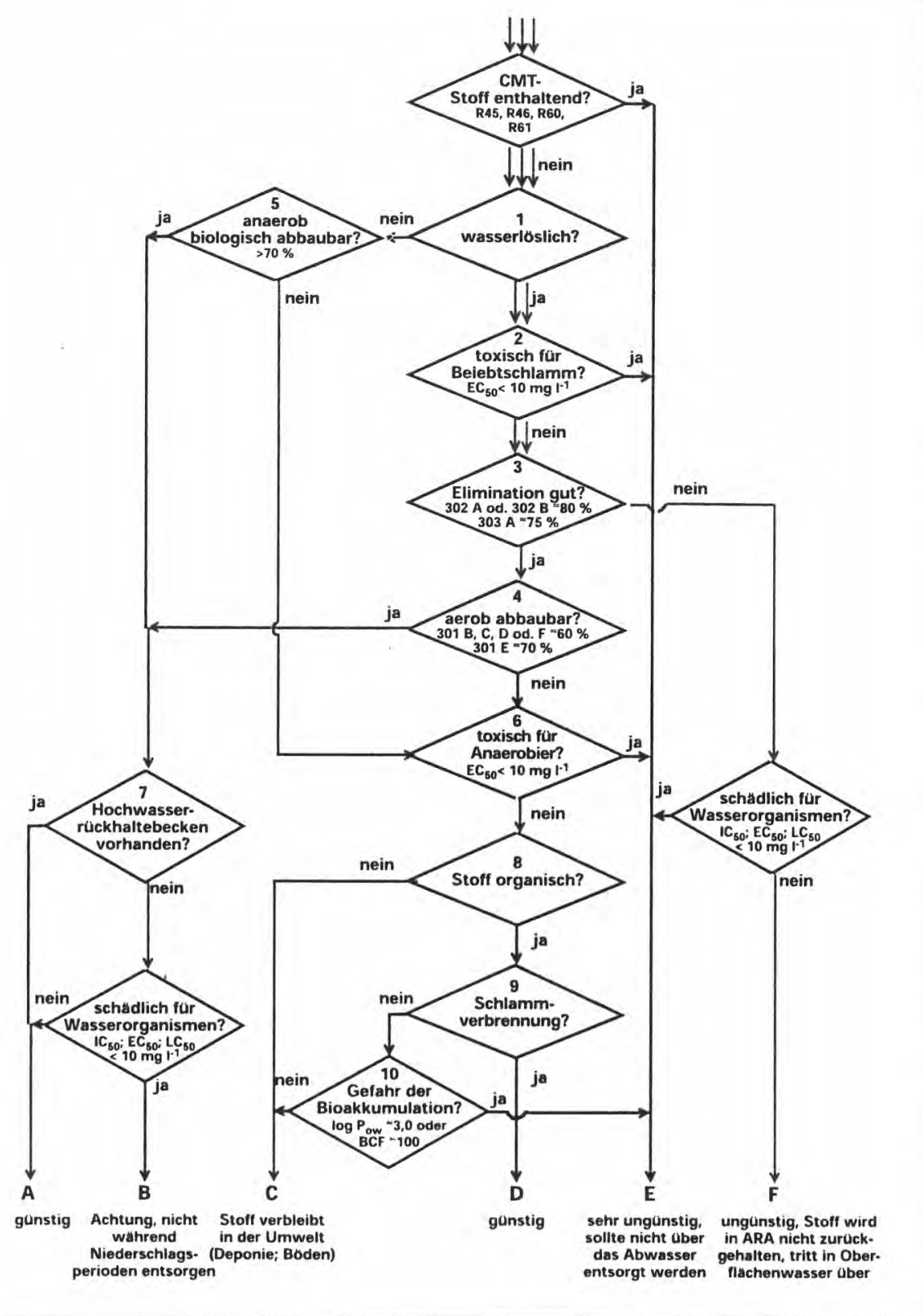

Abb. 6. Klassifizierung der drei fiktiven Substanzen aus Tabelle 4; rot: Beispiel 1, grün: Beispiel 2, blau: Beispiel 3.

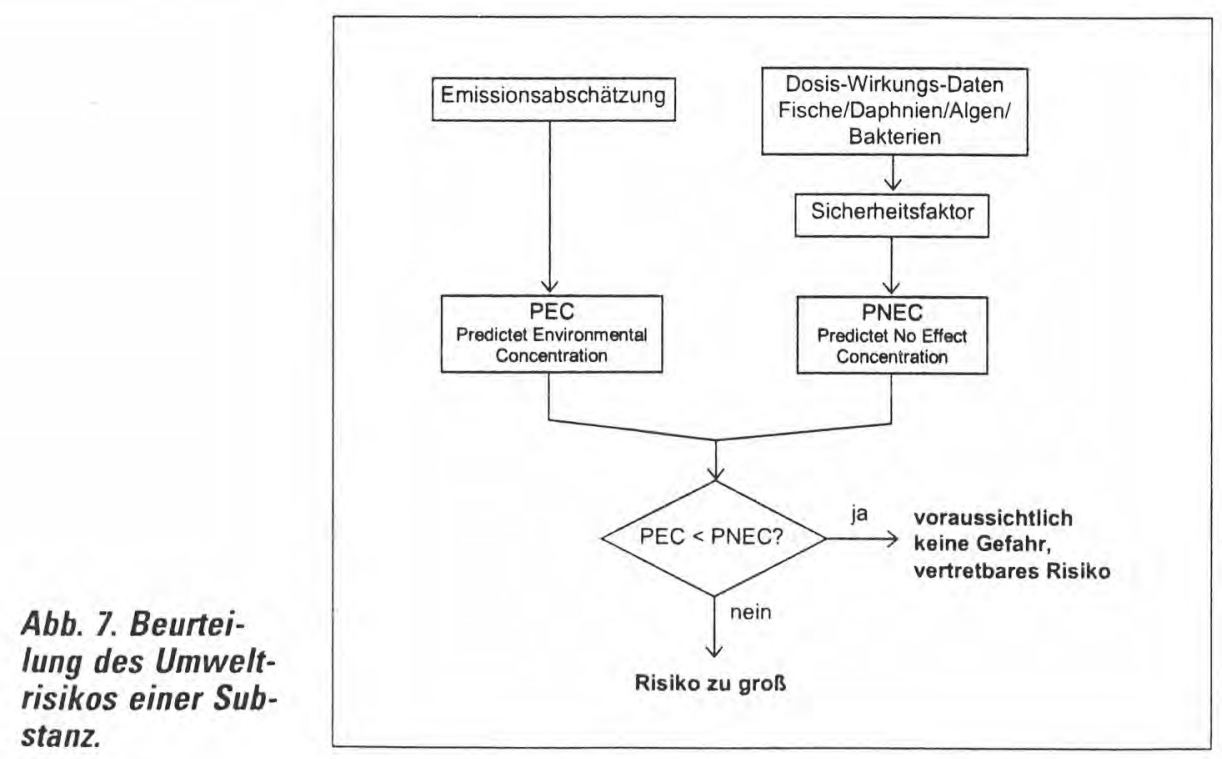

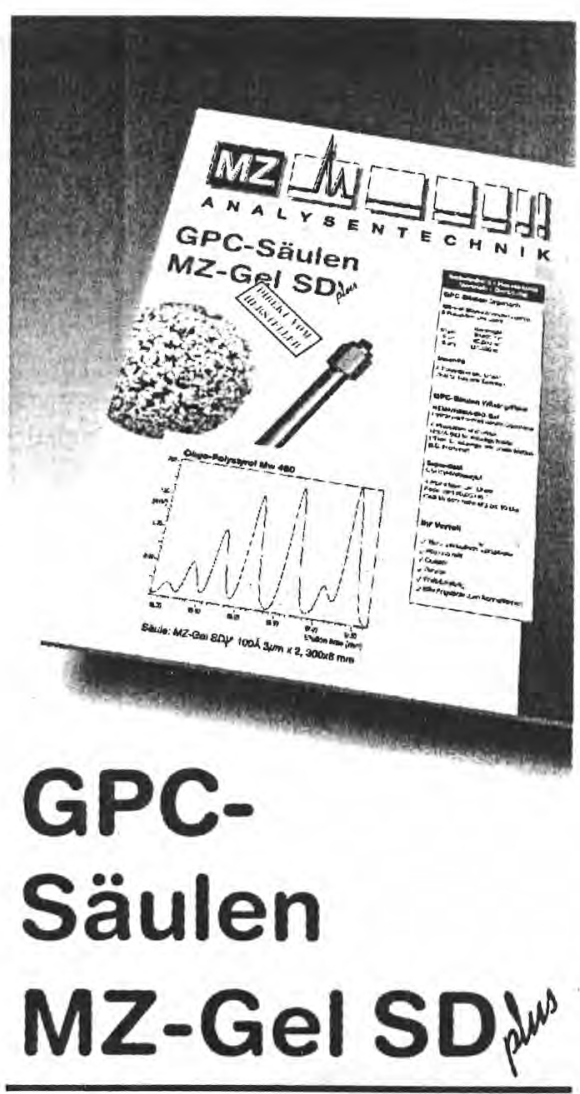

Styroldivinylbenzol

Organisch

6 Porositäten und Linear Korngröße Bodenzahl $10 \mu \mathrm{m} \quad 60.000 \mathrm{~m}^{-1}$ $5 \mu \mathrm{m} \quad 80.000 \mathrm{~m}^{-1}$ $3 \mu \mathrm{m}>100.000 \mathrm{~m}^{-1}$

- Ihr Vorteil

$\checkmark$ micro $\cdot$ analytisch - präparativ $\checkmark$ neu und refill

$\checkmark$ Qualität

$\checkmark$ Service

$\checkmark$ Preis/Leistung

tolle Angebote zum Kennenlemen

T Bitte erkundigen Sie sich bei uns!

\section{Entwicklung • Herstellung} Vertrieb • Beratung

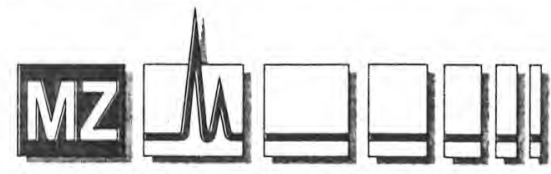

A N A L Y S EN T E C H N I K

Wöhlerstraße 2-6

D-55120 Mainz

e-mail: mz-at@t-online.de

Tel.: 06131/68 6619 http:/iwww.mz-at.de Fax: 06131/68 6620 